\title{
Building muscle, browning fat and preventing obesity by inhibiting myostatin
}

\author{
N. K. LeBrasseur
}

Received: 8 September 2011 / Accepted: 4 October 2011 /Published online: 6 November 2011

(C) Springer-Verlag 2011

\begin{abstract}
The obesity epidemic is an overwhelming global health concern. Interventions to improve body weight and composition aim to restore balance between nutrient intake and energy expenditure. Myostatin, a powerful negative regulator of skeletal muscle mass, has emerged as a potential therapeutic target for obesity and type 2 diabetes mellitus because of the prominent role skeletal muscle plays in metabolic rate and insulin-mediated glucose disposal. In fact, inhibition of myostatin by genetic manipulation or pharmacological means leads to a hypermuscular and very lean build in mice. The resistance of myostatin-null mice to diet-induced obesity, fat mass accumulation and metabolic dysfunction has been presumed to be a result of their large skeletal muscle mass; however, in this issue of Diabetologia, Zhang et al. (doi:10.1007/s00125-011-2304-4) provide evidence that myostatin inhibition also significantly impacts the phenotype of white adipose tissue (WAT). The authors reveal elevated expression of key metabolic genes of fatty acid transport and oxidation and, intriguingly, the presence of brown adipose tissue-like cells in WAT of myostatin-null mice. They also show that pharmacological inhibition of myostatin replicates several of the protective benefits
\end{abstract}

N. K. LeBrasseur $(\bowtie)$

Robert \& Arlene Kogod Center on Aging, Mayo Clinic,

200 First Street SW,

Rochester, MN 55905, USA

e-mail: lebrasseur.nathan@mayo.edu

N. K. LeBrasseur

Department of Physical Medicine \& Rehabilitation, Mayo Clinic,

Rochester, MN, USA

N. K. LeBrasseur

Department of Physiology \& Biomedical Engineering,

Mayo Clinic,

Rochester, MN, USA conveyed by its genetic inactivation. Herein, these data, areas in need of further investigation and the evidence that implicates myostatin as a target for obesity and type 2 diabetes mellitus are discussed.

Keywords ActRIIB - Adipogenesis - Brown fat - Diabetes · Fatty acid oxidation $\cdot$ Myostatin $\cdot$ Skeletal muscle

\begin{tabular}{ll}
\multicolumn{2}{l}{ Abbreviations } \\
ActRIIB & Activin type IIB receptor \\
AMPK & AMP-activated protein kinase \\
BAT & Brown adipose tissue \\
sActRIIB & Soluble decoy activin type IIB receptor \\
UCP & Uncoupling protein \\
WAT & White adipose tissue
\end{tabular}

Over two billion adults worldwide are obese or overweight [1]; a statistic that reflects the widespread and fundamental imbalance between energy consumed and energy expended. Exercise is an effective and beneficial means to promote energy utilisation in the battle against obesity [2]. However, adoption of a more physically active lifestyle, much like improved dietary habits, remains poor and underscores the need for alternative interventions. In this issue of Diabetologia, Zhang and colleagues offer additional support for myostatin inhibition as a potential strategy to counter obesity, and reveal an intriguing new mechanism by which it enhances energy expenditure in mice [3].

Myostatin and the impressive 'double-muscled' phenotype of myostatin-null mice were discovered by the laboratory of Dr Se-Jin Lee in 1997 [4]. Myostatin (also known as growth and differentiation factor 8 [GDF-8]) is a member of the TGF- $\beta$ superfamily. It is predominantly produced in skeletal muscle where it actively suppresses 
cell growth and differentiation by signalling through the activin type IIB receptor (ActRIIB). Myostatin has been highly conserved across evolution [5], and naturally occurring loss-of-function mutations augment skeletal muscle growth in multiple species, including humans [6]. As a result, there has been great interest in developing inhibitors of myostatin (e.g. antibodies, soluble decoy ActRIIB [sActRIIB] and propeptides) to build and/or regenerate skeletal muscle in the face of ageing (sarcopenia) and disease (cachexia and degeneration) (for examples, see [7-11]).

Skeletal muscle is the primary site of insulinmediated glucose disposal, the largest reservoir of glycogen in the human body and a key determinant of energy expenditure. Hence, several recent studies have also investigated the effects of genetic and pharmacological inhibition of myostatin, and the resultant 'resistance exercise-trained' phenotype, on the prevention and treatment of obesity and type 2 diabetes mellitus (reviewed in $[12,13])$. Similar to these reports, Zhang and colleagues demonstrate that inhibition of myostatin increases skeletal muscle mass and prevents accumulation of body weight, fat mass, and circulating concentrations of triacylglycerol caused by high-fat feeding [3]. However, while earlier studies have universally concluded these benefits were secondary to the increase in skeletal muscle, Zhang et al. show remarkable changes in the adipose tissue of myostatin-null mice. Specifically, the authors demonstrate that compared with wild-type mice, white adipose tissue (WAT) of myostatin-deficient mice more highly expresses key genes of lipid transport, synthesis, hydrolysis and oxidation. They also measured an increase in the expression of genes encoding transcription factors and uncoupling proteins (UCP) that are more common to brown adipose tissue (BAT). In fact, histological analysis of WAT explants derived from myostatin-deficient mice revealed the presence of BAT-like cells, defined by their relatively small diameter, inclusion of multilocular lipid droplets and positive staining for UCP-1. A phenotypic shift of WAT towards its more metabolically active counterpart, BAT, is now commonly referred to as 'browning' or 'beiging'. This phenomenon is of particular interest to the fields of obesity and type 2 diabetes mellitus because BAT is a powerful energy-burning and heat-producing tissue that has recently been confirmed to be present and active in adult humans [14-16]. Of note, Zhang et al. report myostatin-null mice were $\sim 1^{\circ} \mathrm{C}$ warmer than wild-type mice, and WAT explants (as well as liver explants and cultures of primary myotubes) from myostatin-null mice demonstrated higher rates of fatty acid oxidation than wild-type mice [3]. Collectively, these data suggest inactivation of myostatin protects mice from diet-induced obesity not only because of increased energy utilisation by a larger mass of skeletal muscle, but also as a result of enhanced metabolic activity in WAT and potentially in other tissues.

Importantly, from a therapeutic perspective, the authors also show that pharmacological inhibition of myostatin replicates several of the protective benefits conveyed by its genetic inactivation. In the present study, treatment of mice with sActRIIB $(5 \mathrm{mg} / \mathrm{kg}$, three times per week) concurrent with 12 weeks of high-fat feeding prevented the significant increases in body weight, WAT depot weights and circulating triacylglycerols observed in mice receiving vehicle. These data corroborate those of Akpan et al. from a study in which high-fat fed mice receiving sActRIIB for 10 weeks also gained less fat mass and had lower glucose and cholesterol concentrations compared with vehicletreated mice [17]. Zhang and colleagues also observed that administration of SActRIIB prevented the significant downregulation of key genes of uncoupling and oxidative metabolism in WAT that was observed in vehicle-treated mice in response to high-fat feeding. While ActRIIB binds a broad repertoire of ligands, the similar phenotype between myostatin-null and sActRIIB-treated mice suggests inhibition of myostatin largely accounts for the benefits observed. These data support the notion that myostatin blockade may be a viable strategy to prevent obesity in humans (illustrated in Figs 1 and 2). Whether or not pharmacological inhibition of myostatin can also attenuate the progression of or reverse obesity remains to be determined.

It is plausible that myostatin inhibition improves the gene expression profile and metabolic state of WAT through direct and/or indirect mechanisms. With regard to a direct mechanism, the prevention of myostatin and other ActRIIB ligands from eliciting signals in adipocytes, preadipocytes and/or mesenchymal stem cells may have altered gene expression and/or cell differentiation. In a very recent publication in Diabetologia, the authors of the current study reported an increased abundance and activity of the fuel-sensing enzyme AMP-activated protein kinase (AMPK) in the adipose tissue, liver and skeletal muscle of mice lacking myostatin [18]. While it is unclear how the absence of myostatin stimulates AMPK signalling, it is a mechanism that would attenuate fatty acid synthesis and promote fatty acid oxidation in WAT (reviewed in [19]). However, the role of myostatin in adipogenesis is controversial and the direct effects of myostatin inhibition on adipogenesis have not been thoroughly studied. Moreover, a serious challenge to the hypothesis that myostatin inhibition directly affects WAT comes from an elegant study that assessed whether targeted expression of a dominant negative ActRIIB in adipose tissue would prevent dietinduced obesity and metabolic dysfunction [20]. The 


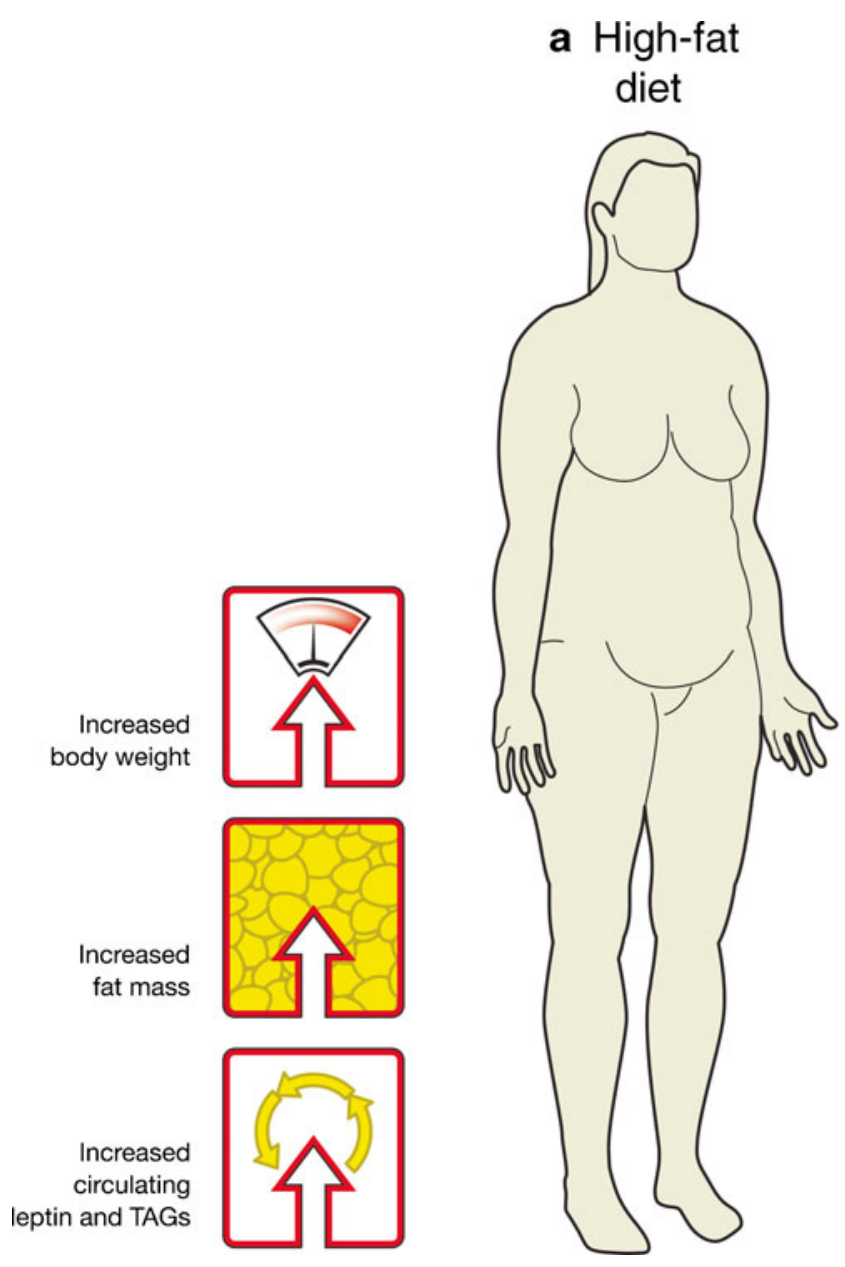

Fig. 1 Myostatin inhibition may be a strategy to prevent diet-induced changes in body weight and body composition. High-fat feeding increases body weight, fat mass and circulating concentrations of leptin and triacylglycerols (TAGs) (illustrated in a). In mice, Zhang and colleagues demonstrate that inhibition of myostatin signalling, through delivery of a soluble ActRIIB decoy receptor (sActRIIB),

results convincingly showed that there were no beneficial effects of inhibiting myostatin signalling in adipose tissue on weight gain, fat mass, adipocyte size, glucose homeostasis or circulating leptin or triacylglycerol concentrations. In contrast, muscle-specific expression of dominant negative ActRIIB improved all of these variabless of body composition and metabolism, suggesting that the adaptations in WAT phenotype in response to myostatin inhibition are primarily indirect and secondary to changes in skeletal muscle. A growing body of evidence highlights skeletal muscle as a prominent node in the powerful and complex cross-talk between metabolically active tissues. For instance, mice homozygous for a mutation in the myostatin gene are resistant to diet-induced hepatic steatosis, elevations in inflammatory cytokines and macrophage infiltration/activation in adipose tissue and skeletal muscle compared with wild-type and heterozygous mice [21]. Postnatal blockade of

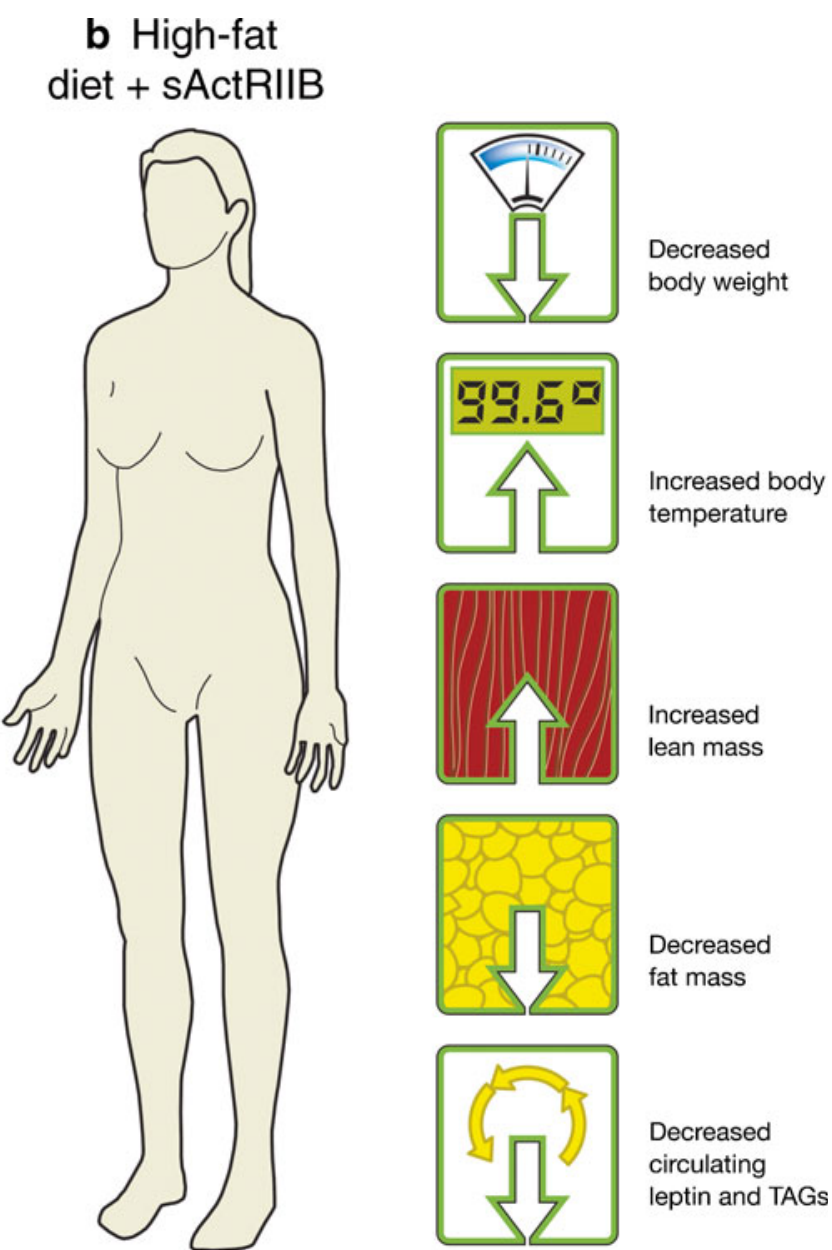

increases skeletal muscle mass and body temperature and minimises the deleterious effects of nutrient excess. As illustrated in $\mathbf{b}$, their findings raise the possibility that in humans, pharmacological inhibition of myostatin and potentially other ActRIIB ligands may prevent the development of obesity

myostatin with a neutralising antibody in obese insulin-resistant mice significantly improved glucose homeostasis, lowered circulating triacylglycerols and increased circulating concentrations of the adipose tissue-derived cytokine, adiponectin [17, 22]. The absence of myostatin also appears to protect high-fatfed LDL receptor-null mice from dyslipidaemia and atherogenesis, as LDL receptor/myostatin double knockout mice have decreased VLDL generation, improved lipid profiles and reduced atherogenesis progression [23]. In view of the coordinated responses of muscle, liver, adipose tissue and the vasculature highlighted in these models, it is likely that the benefits of myostatin inhibition on WAT gene expression and metabolic activity observed by Zhang et al. are predominantly indirect. Nonetheless, the significant effect of myostatin inhibition on the phenotype of WAT deserves further study. The 


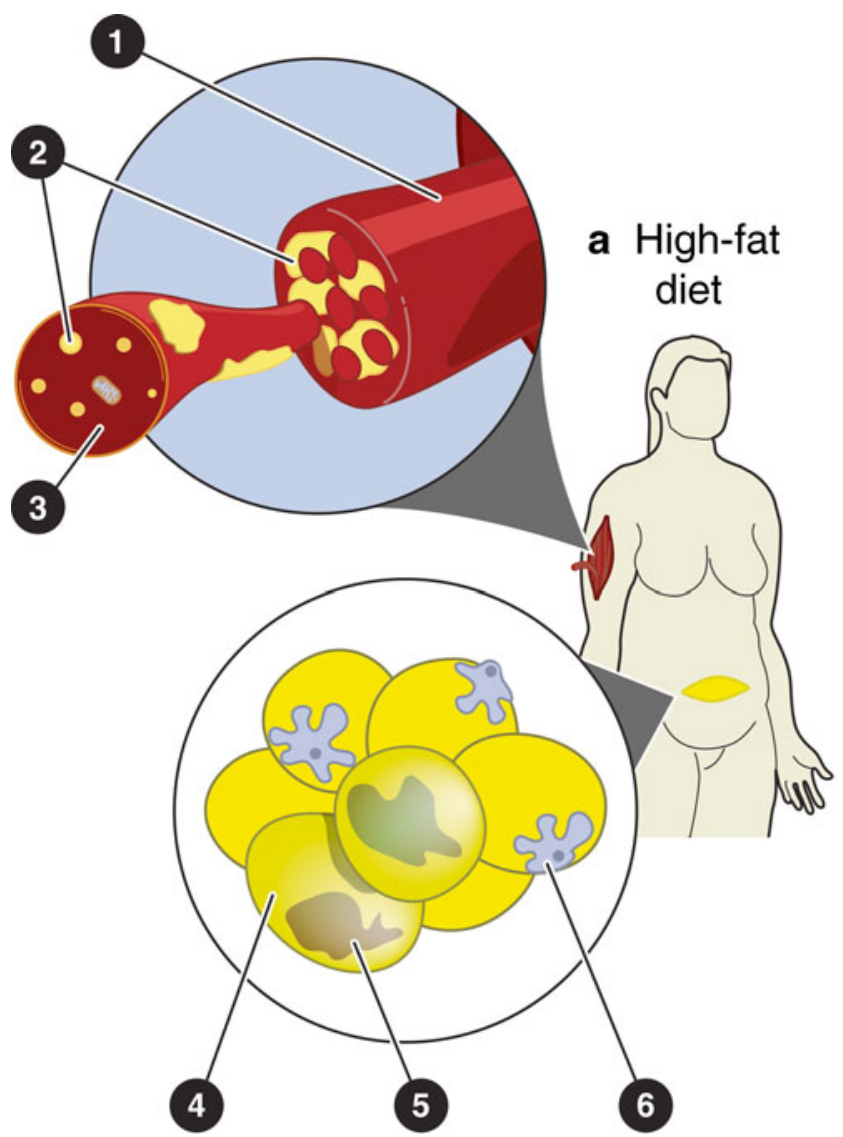

Fig. 2 Inhibition of myostatin improves the metabolic state of skeletal muscle and adipose tissue. Diet-induced obesity leads to alterations in the quantity and quality of skeletal muscle (top insets) and adipose tissue (bottom insets). While several studies have shown that myostatin inhibition leads to an increase in skeletal muscle mass and fibre size (1) (untreated vs treated with sActRIIB), Zhang et al. provide additional evidence in mice that suggest myostatin inhibition may confer salutary effects on the metabolic state of skeletal muscle and adipose tissue in humans. As illustrated, myostatin inhibition may abrogate the accumulation of extra- and intramyocellular lipids (2) and promote fatty acid oxidation in skeletal muscle by preventing reductions in the expression, abundance and activity of key mediators of oxidative metabolism caused by high-fat feeding (3). Their data

possibility of a network of hormonal communication (e.g. myokines) between skeletal muscle and adipose tissue, as well as other metabolically important organs, is of particular interest.

From a clinical perspective, there is a growing body of clinical evidence to support myostatin blockade as a therapeutic strategy for obesity and type 2 diabetes mellitus. For example, increased myostatin production and, more impressively, myostatin secretion has been observed in skeletal muscle and adipose tissue samples derived from obese and extremely obese women. Increased circulating levels of myostatin in this cohort were found to be correlated with insulin resistance [24]. In addition, the expression of the gene encoding myostatin

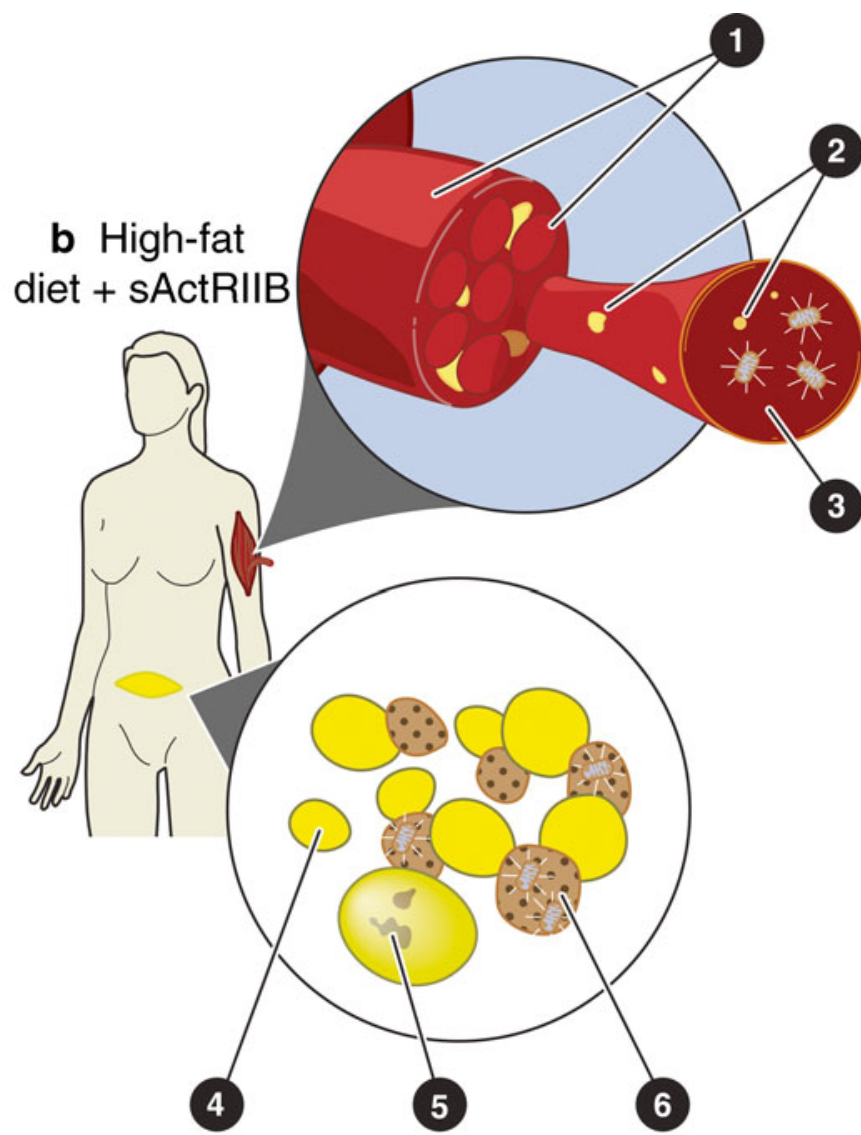

also imply that diet-induced adipocyte hypertrophy (4), accumulation of triacylglycerols (5) and infiltration of macrophages (6, illustrated in a) in adipose tissue may be prevented by myostatin inhibition. Similar to skeletal muscle, these salutary effects of inhibiting myostatin appear to be mediated by the maintained expression, abundance and activity of key mediators of fatty acid transport and oxidation in adipose tissue in response to high-fat feeding. Intriguingly, Zhang and colleagues also provide evidence that myostatin inhibition drives the 'browning' of WAT in mice, meaning, the appearance of energy consuming, mitochondrial-rich and thermogenic brown fat-like cells (6, illustrated in b). Collectively, these data obtained in mice provide further support for myostatin inhibition as a potential means to prevent obesity and type 2 diabetes mellitus in humans

$(M S T N)$ in skeletal muscle of obese patients undergoing gastric bypass surgery was significantly decreased in response to weight loss and associated with improved insulin action $[25,26]$. Increased expression of MSTN was also recently detected in skeletal muscle biopsies of healthy but at risk first-degree relatives of patients with type 2 diabetes mellitus [27]. Collectively, these clinical observations suggest that myostatin may contribute to deleterious changes in body weight, body composition and whole-body metabolism. Based on previous studies referred to in this commentary and new insights from Zhang and colleagues, myostatin inhibition may be a strategy to enhance energy expenditure in the battle against obesity and type 2 diabetes mellitus. 
Acknowledgements The author would like to thank Gunther Chanange from the Mayo Clinic Center for Innovation, for creating the illustrations in this manuscript. This author would also like to acknowledge the support of Mayo Clinic and a generous gift from Robert and Arlene Kogod.

Contribution statement NK LeBrasseur was responsible for the conception, writing and final approval of this article.

Duality of interest The author declares that there is no duality of interest associated with this manuscript.

\section{References}

1. Finucane MM, Stevens GA, Cowan MJ et al (2011) National, regional, and global trends in body-mass index since 1980: systematic analysis of health examination surveys and epidemiological studies with 960 country-years and 91 million participants. Lancet 377:557-567

2. Shaw K, Gennat H, O'Rourke P, Del Mar C (2006) Exercise for overweight or obesity. Cochrane Database Syst Rev, Issue 4. Art. no.:CD003817. doi:10.1002/14651858.CD003817.pub3

3. Zhang C, McFarlane C, Lokireddy $S$ et al (2011) Inhibition of myostatin protects against diet-induced obesity through enhancing fatty acid oxidation and promoting brown adipose phenotype. Diabetologia. doi:10.1007/s00125-011-2304-4

4. McPherron AC, Lawler AM, Lee SJ (1997) Regulation of skeletal muscle mass in mice by a new TGF-beta superfamily member. Nature 387:83-90

5. McPherron AC, Lee S-J (1997) Double muscling in cattle due to mutations in the myostatin gene. Proc Natl Acad Sci 94:12457-12461

6. Schuelke M, Wagner KR, Stolz LE et al (2004) Myostatin mutation associated with gross muscle hypertrophy in a child. $\mathrm{N}$ Engl J Med 350:2682-2688

7. LeBrasseur NK, Schelhorn TM, Bernardo BL, Cosgrove PG, Loria PM, Brown TA (2009) Myostatin inhibition enhances the effects of exercise on performance and metabolic outcomes in aged mice. J Gerontol A Biol Sci Med Sci 64:940-948

8. Zhou X, Wang JL, Lu J et al (2010) Reversal of cancer cachexia and muscle wasting by ActRIIB antagonism leads to prolonged survival. cell 142:531-543

9. Bogdanovich S, Krag TOB, Barton ER et al (2002) Functional improvement of dystrophic muscle by myostatin blockade. Nature 420:418-421

10. Zimmers TA, Davies MV, Koniaris LG et al (2002) Induction of cachexia in mice by systemically administered myostatin. Science 296:1486-1488

11. Wagner KR, Liu X, Chang X, Allen RE (2005) Muscle regeneration in the prolonged absence of myostatin. Proc Natl Acad Sci U S A 102:2519-2524
12. Allen DL, Hittel DS, McPherron AC (2011) Expression and function of myostatin in obesity, diabetes, and exercise adaptation. Med Sci Sports Exerc 43:1828-1835

13. LeBrasseur NK, Walsh K, Arany Z (2011) Metabolic benefits of resistance training and fast glycolytic skeletal muscle. Am J Physiol Endocrinol Metab 300:E3-E10

14. Cypess AM, Lehman S, Williams G et al (2009) Identification and importance of brown adipose tissue in adult humans. N Engl $\mathrm{J}$ Med 360:1509-1517

15. Virtanen KA, Lidell ME, Orava J et al (2009) Functional brown adipose tissue in healthy adults. N Engl J Med 360:1518-1525

16. van Marken Lichtenbelt WD, Vanhommerig JW, Smulders NM et al (2009) Cold-activated brown adipose tissue in healthy men. $\mathrm{N}$ Engl J Med 360:1500-1508

17. Akpan I, Goncalves MD, Dhir R et al (2009) The effects of a soluble activin type IIB receptor on obesity and insulin sensitivity. Int J Obes (Lond) 33:1265-1273

18. Zhang C, McFarlane C, Lokireddy S et al (2011) Myostatindeficient mice exhibit reduced insulin resistance through activating the AMP-activated protein kinase signalling pathway. Diabetologia 54:1491-1501

19. Ruderman NB, Saha AK, Kraegen EW (2003) Minireview: malonyl CoA, AMP-activated protein kinase, and adiposity. Endocrinology 144:5166-5171

20. Guo T, Jou W, Chanturiya T, Portas J, Gavrilova O, McPherron AC (2009) Myostatin inhibition in muscle, but not adipose tissue, decreases fat mass and improves insulin sensitivity. PLoS One 4:e4937

21. Wilkes JJ, Lloyd DJ, Gekakis N (2009) Loss-of-function mutation in myostatin reduces tumor necrosis factor alpha production and protects liver against obesity-induced insulin resistance. Diabetes 58:1133-1143

22. Bernardo BL, Wachtmann TS, Cosgrove PG et al (2010) Postnatal PPARdelta activation and myostatin inhibition exert distinct yet complimentary effects on the metabolic profile of obese insulinresistant mice. PLoS One 5:e11307

23. Tu P, Bhasin S, Hruz PW et al (2009) Genetic disruption of myostatin reduces the development of proatherogenic dyslipidemia and atherogenic lesions in $L d l r$ null mice. Diabetes 58:1739-1748

24. Hittel DS, Berggren JR, Shearer J, Boyle K, Houmard JA (2009) Increased secretion and expression of myostatin in skeletal muscle from extremely obese women. Diabetes 58:30-38

25. Milan G, Dalla Nora E, Pilon C et al (2004) Changes in muscle myostatin expression in obese subjects after weight loss. J Clin Endocrinol Metab 89:2724-2727

26. Park JJ, Berggren JR, Hulver MW, Houmard JA, Hoffman EP (2006) GRB14, GPD1, and GDF8 as potential network collaborators in weight loss-induced improvements in insulin action in human skeletal muscle. Physiol Genomics 27:114-121

27. Palsgaard J, Brons C, Friedrichsen M et al (2009) Gene expression in skeletal muscle biopsies from people with type 2 diabetes and relatives: differential regulation of insulin signaling pathways. PLoS One 4:e6575 